\title{
Local distribution of cobalt and selective enrichment of platinum in oceanic ferromanganese nodules
}

\author{
Dmitriy Belyanin ${ }^{1,2, *}$, Sergey Zhmodik $^{1,2}$, Evgeniya Airiyants ${ }^{1}$, and Ivan Kirichenko ${ }^{1}$ \\ ${ }^{1}$ Sobolev Institute of Geology and Mineralogy SB RAS, 630090, Novosibirsk, Koptug ave., 3, Russia \\ ${ }^{2}$ Novosibirsk State University, 630090, Novosibirsk, Pirogova st., 1, Russia
}

\begin{abstract}
The distribution of cobalt in the structure of ferromanganese nodules (FMN) was studied using beta-autoradiography. In some cases, the content of element increased in the outer part of the nodules, or in thin layers within the nodules. Layered type of cobalt distribution in FMN indicates changing conditions in deposition during their formation, in particular also the living conditions of microorganisms known to inhabit oceanic ferromanganese nodules. Experimental studies suggested that colonies of micromycetes living at the surface of the nodules can have an important impact on the selective accumulation of platinum and other elements in FMN.
\end{abstract}

\section{Introduction}

It is known that oceanic ferromanganese nodules (FMN) concentrate significant amounts of trace elements like nickel, copper and cobalt (which are main ore metals in FMN) depending on their conditions of formation. The content of these elements is influenced by diagenetic, hydrogenic and biogenic factors. The goal of our work was to study the distribution of elements in hydrogenic ferromanganese nodules. Of particular interest was the distribution of precious and ore metals including cobalt and platinum. Since directly determining the distribution of platinum in nodules by autoradiography did not allow the imaging of their zonation in ferromanganese shell, an experiment was conducted on the interaction of FMN and solution containing platinum. Our hypotheses were that the FMN can scavenge the platinum, and that microorganisms might be responsible for enrichment and zonation of $\mathrm{Pt}$ and other trace elements in the FMN.

\section{Samples and method}

The studied ferromanganese nodules were taken from a collection of oceanic ferromanganese crusts and nodules kindly provided by Prof. Victor Sharapov, who participated in the 8th cruise of research vessel "Akademik Vinogradov" to the ClarionClipperton Zone of the Pacific Ocean in 1986. Dredging was conducted at 4,000-5,400 m

\footnotetext{
*Corresponding author: bel@igm.nsc.ru
} 
depth from the bottom to the top of a narrow ridge in the southern part of the Clarion fracture (Lat 16 $16^{\prime}-16^{\circ} 20^{\prime} \mathrm{N}$; Long $136^{\circ} 46^{\prime}-136^{\circ} 57^{\prime} \mathrm{W}$ ).

Local spatial distribution of elements was studied in thin sections of the nodule by SRXRF (SSTRC, INP SB RAS) and autoradiography (IGM SB RAS). Experimentally FMN was reacted with a solution containing platinum using a radioactive label $\left({ }^{195 \mathrm{mPt}},{ }^{193 \mathrm{~m} P t}\right.$, ${ }^{199} \mathrm{Au}$ and ${ }^{192} \mathrm{Ir}$ ). The results of the distribution of platinum were recorded on betaautoradiography. The activities of gamma-radiation substances (solution of sea salt before and after experiment, microfungi colony) participating in the experiment and irradiated sections of FMN were determined at the facility for neutron activation analysis. Scanning electron microscope (SEM) was used to determine the mineralogical characteristics of the structural features of FMN allotted to autoradiography analysis.

\section{The local distribution of elements}

Irregular spatial distribution of the elements Sm, La, and As in FMN can be seen in the early expositions. These elements in the core portion are reduced significantly as compared to the ore shell. The core region shows a sudden drop of the blackening density on autoradiography images determined through contrast changes in the concentrations of elements (radionuclides) in inclusions of the rock fragments in the ore shell. In addition, the density distribution of elements in the slice of FMN shown on autoradiogram reflects concentric zonation and, in some cases, the radial structure of the nodules.

An intriguing late radiography was obtained after cooling the sample for a long period (750 days), which records the spatial distribution of one of the most interesting elements of iron-manganese ore - cobalt. High cobalt content in the peripheral parts of some FMN were found. Significant darkening of the outer part of the nodules (3-5 $\mathrm{mm}$ from the edge) are indicated on autoradiography of these samples (Fig. 1). In some cases on the very edge there is a thin rim width $(0.5 \mathrm{~mm})$, that indicate a zone of enrichment-depletion of cobalt edge portion of the ferromanganese nodules.
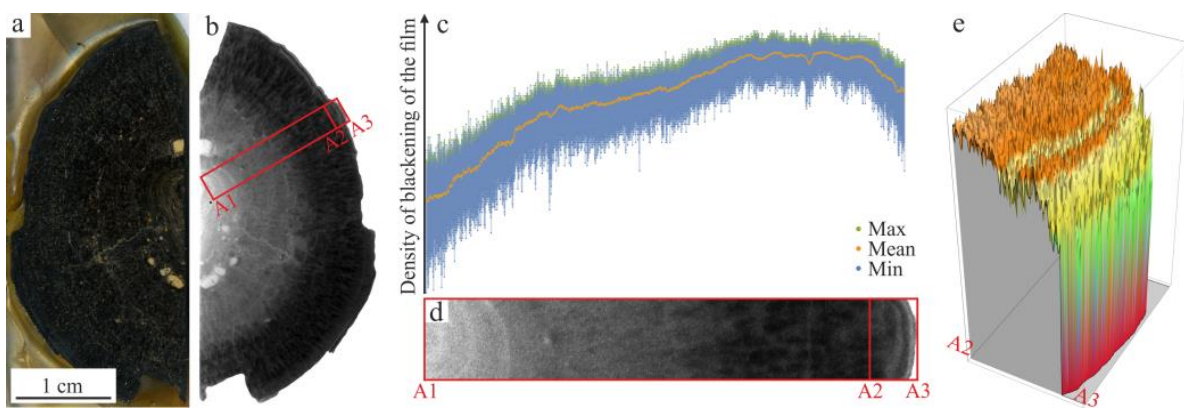

Fig. 1. Thin section of the nodule (a); autoradiography images (b) showing the reduction of cobalt content from the edge to center of ferromanganese nodules. Higher magnification (d) and graphs of the density of blackening of the emulsion (c, e) reveals a double rim in the outer part of ferromanganese nodules.

In the sample with a distinct change in the distribution of cobalt, concentration profiles were determined by means of scanning electron microscopy. In the outer part, the cobalt content was equal to $0.6-1$ wt. $\%$, and in the central part $0.2-0.3$ wt. $\%$. Thus, using the logarithmic dependence of the density of blackening of the film on the concentration of the radiating element allows the opportunity to link observed autoradiography image density to the content of cobalt (Fig. 2) [1]. A particular feature of this sample is the presence of thin interlayers of argillaceous (alumino-silicate) matter between a wide outer rim with high content of metal and the rest. Data on the distribution of cobalt in the nodules section 
obtained by means of SR-XRF were compared with autoradiography images showing the distribution of cobalt on the plate sawed parallel in the same sample. On both plates, the largest difference in the distribution of metals is created by the transition of iron-manganese - aluminum silicate substance. Overall, the data on the distribution of elements studied are well correlated.
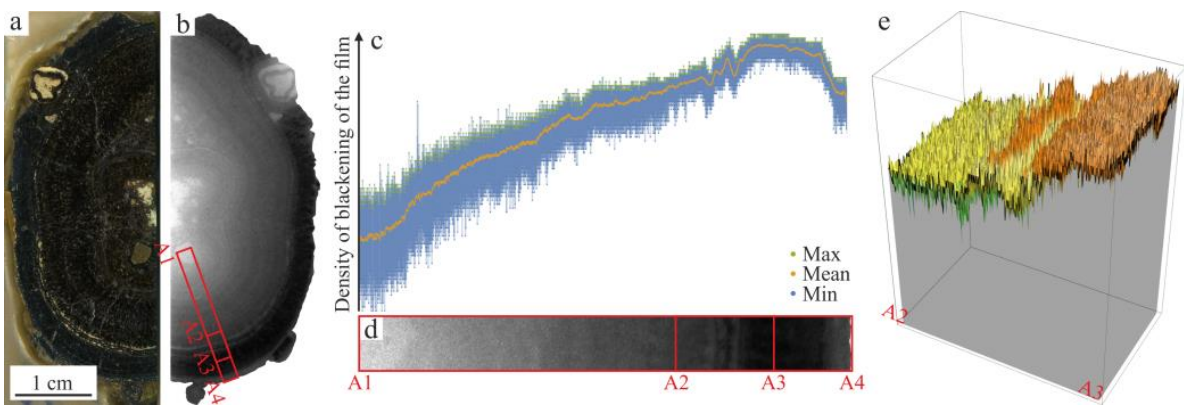

Fig. 2. Thin section of the nodule (a); enrichment of cobalt manifested by autoradiography in outer edges of nodules, separated from the rest of interlayers of clayey matter (b). At higher magnification (d), it is noticeable that the clayey layers themselves are depleted in cobalt relative to iron-manganese substances, as also by the graphs of the density of blackening of the emulsion (c, e).

\section{The experiment on interaction of FMN and Pt solutions}

For a study of the process of absorption of platinum by the substance of FMN, we used thin sections of FMN and $35 \%$ solution of sea salt containing $3.03 \mathrm{mg} / \mathrm{l}$ of chloride of platinum. Before transfer to the solution, the platinum was irradiated by neutron flux, allowing to determine its relative concentration in the system by daughter radioisotopes $195 \mathrm{mPt}, 193 \mathrm{mPt}$ and 199Au, which are beta and gamma emitters. Local spatial distribution of platinum scavenged from the solution on the surface of FMN thin sections was detected with the beta-autoradiography method. For determining the activity and the relative concentration of radionuclides in the system, we used the method of high-resolution gamma-ray spectrometry.

After the experiment on the interaction with seawater containing $\mathrm{Pt}$, the autoradiograms showed the spatial distribution of $\mathrm{Pt}$ on cross section of nodules. An heterogeneous distribution of $\mathrm{Pt}$ with a maximum enrichment along small cracks, micro inclusions, and in some cases the marginal parts of the nodules was observed. In addition, a concentriclayered distribution of Pt-enrichment layers was clearly visible consisting mainly of Fe and Mn hydroxides (with a minimum amount of Al-Si component).

During the Pt experiment in solution with nodules, we observed the growth of colonies of micro-organisms - microfungi. Autoradiography showed that they actively absorbed platinum from a solution. Heterogeneities in the distribution of platinum determined in the structure of the colony were found, in particular linear anomalies corresponding to the internal structure and an increase in the concentration at the edges of the colonies.

Notably, in similar experiments on the deposition of platinum from seawater on the surface of thin sections of sulfide minerals and ores, such colonies of microorganisms did not occur. However, these samples of minerals were selected from gold ore deposits of Siberia (Baley, Zun-Holba, Sukhoi Log, and others), not from seawater deposits.

Intriguingly, after irradiation of a plate of platinum used for preparation of standards $(99.99 \% \mathrm{Pt})$, it showed an impurity of iridium. Absorption of microorganisms from seawater on this standard plate of platinum showed marked selectivity of uptake of platinum in relation to the iridium impurity. The daughter isotope activities of platinum and 
iridium in the colonies of fungi were more than an order of magnitude higher compared to the nodules and 200 times more than ratio in the initial solution. Thereby the standard of platinum was cleaned from iridium impurities $-\mathrm{n} \cdot 10^{-4} \mathrm{wt} \%$ to $10^{-5}-10^{-6} \mathrm{wt} \%$, that is by 1.5-2 orders of magnitude.

The samples of microfungi were studied by SEM. The images show that the body of fungi colony consists of many interwoven hyphae with a diameter of $1-10$ microns. The density of these filaments in the colonies and their size is not constant. In addition, in many places they contain grape-like clusters of spores (conidia). High platinum content at the level of a few percent were established in all the colonies of micromycetes. Thus, platinum concentration from initial solution is enriched by 10,000 times in fungi colonies (Fig. 3) [2].
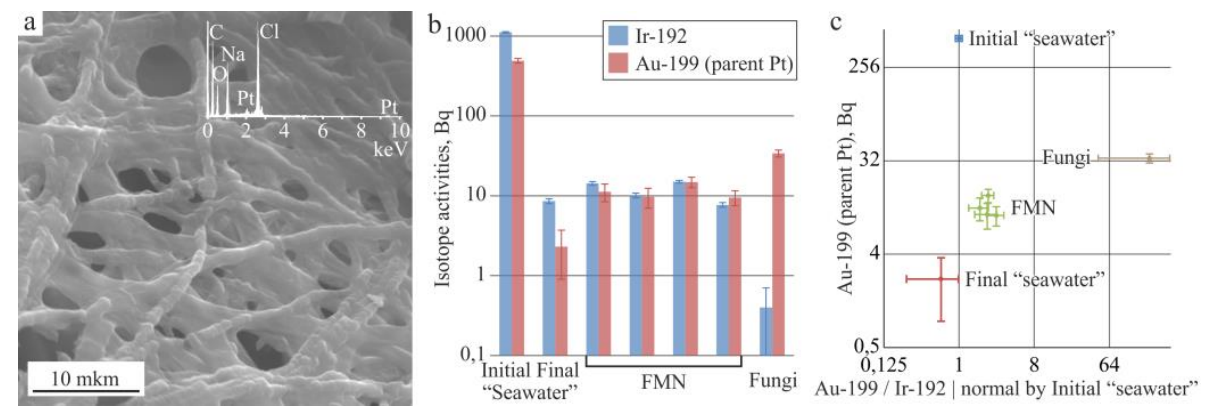

Fig. 3. Secondary electron image of platinum enriched mycelium (a), and selectivity of uptake of platinum in relation to impurity iridium from solution of sea salt by fungi and FMN (b, c).

Seeding spores of microorganisms with nodule material of Guyot Lamont, Clarion fracture and Magellan Mountains in the nutrient medium (agar-agar)enabled to obtain colonies of fungi and their specific features. In particular, conidia of the fungus are formed in chains that are arranged in clusters or tassels. Morphological and micro-morphological features allow the fungus to be attributed to the genus Penicillium. In addition, a study of colonies of fungi grown on nutrient media showed that they actively concentrate trace elements of the FMN. In the study of the vertical structure of the colony of micromycetes, it was found that the concentration of $\mathrm{Mn}, \mathrm{Cu}, \mathrm{Ni}$ and $\mathrm{Co}$ increases from mycelia to conidia. This behavior may be related to the feeding habits of fungi. They are characterized by osmotrophy type of food - absorption of nutrients across the surface of the mycelium and transport by hyphae, conidiophores the conidia.

\section{Discussion}

FMN acquire metals from two sources, seawater (hydrogenetic) and pore fluids (diagenetic) [3]. The contribution of these sources and resulting characteristics of the structure and composition of nodules are significantly different for various parts of the ocean floor. Also described earlier, presence of layering indicate even changing conditions format the same site such as the Clarion-Clipperton Zone. This can be explained, for example, by fluctuating bioproductivity in the equatorial Pacific surface waters during glacial-interglacial periods which led to changing organic carbon flux to the sediment and changing oxygen consumption in near surface sediments. Furthermore, reduced ventilation of the deep ocean during glacial periods may have led to suboxic conditions in near-surface sediments [4].

There are many reports of bacterial species from manganese nodules $[5,6]$. Minerals in nodules are not only formed by mineralization but also by biologically driven processes involving microorganisms (biomineralization) [7]. Oxidation of $\mathrm{Mn}$ is believed to be controlled by microbial activity [8]. In numerous works, the role of the biogenic factor in the accumulation and isotopic separation of metals in the process of formation of 
ferromanganese crusts and nodules are discussed [9, 10]. The results of our experiments provide additional clues to the biogenic factor paradigm confirming high platinum sorption potential of the physiologically active biomass of microscopic fungi and their cell envelopes (chitin) and thus contributing actively to their scavenging by the FMN [11].

The reported study was funded by RFBR by the research project No. 15-35-21024 and Integration Project SB RAS N 51, and state assignment (No. 0330-2016-0011). Geochemical analyses were carried out at the Analytical Center for Multi-Elemental and Isotope Research, SB RAS and at the Siberian Center of Synchrotron and Terahertz Radiation, SB RAS. The authors are grateful to Profs. Viktor N. Sharapov and Anatoly T. Titov for providing the FMN collection and assistance in performing the SEM analyses. The authors are also very grateful to Profs. Michael Kersten for valuable comments and corrections.

\section{References}

1. D.K. Belyanin, S.M. Zhmodik, I.S. Kirichenko, Radioactivity and radioactive elements in environment. Proceedings of IV international conference, Tomsk, 91-95 (2013)

2. S.M. Zhmodik, D.K. Belyanin, A.G. Mironov, V.S. Parkhomenko, A.T. Titov, T.V. Teplyakova, V.G. Tsimbalist, A.V. Tatarinov, Dokl. Earth Sci. 427, 777-782 (2009)

3. J.R. Hein, K. Mizell, A. Koschinsky, T.A. Conrad, Ore Geol. Rev. 51, 1-14 (2013)

4. A.V. Wegorzewski, T. Kuhn, Mar. Geol. 357, 123-138 (2014)

5. J. Veillette, J. Sarrazin, A.J. Gooday, J. Galéron, J.-C. Caprais, A. Vangriesheim, J. Étoubleau, J.R. Christian, S.K. Juniper, Deep-Sea Res. 54, 11, 1912-1935 (2007)

6. J. Akai, S. Akiyama, A. Tsuchiyama, K. Akai, Phys. Chem. Earth Parts A/B/C, 58-60, 42-48 (2013)

7. X. Wang, W.E.G. Müller, Trends in Biotechnology, 27, 6, 375-383 (2009)

8. M. Villalobos, J. Bargar, G. Sposito, Elements, 1, 4, 223-226 (2005)

9. S.H. Little, D.M. Sherman, D. Vance, J.R. Hein, Earth Planet. Sci. Lett. 396, 213-222 (2014)

10. L.E. Wasylenki, J.W. Swihart, S.J. Romaniello, Geochim. Cosmochim. Acta, 140, 212226 (2014)

11. L.M. Pavlova, V.I. Radomskaya, L.P. Shumilova, A.M. Ionov, V.V. Ivanov, A.V. Poselyuzhnaya, Geochem. Int., 56, 5, 450-461 (2018) 